\title{
Multiscale blood vessel delineation using $B$-COSFIRE filters
}

\author{
Nicola Strisciuglio ${ }^{1,2}$, George Azzopardi ${ }^{1,3}$, Mario Vento $^{2}$, and Nicolai Petkov ${ }^{1}$ \\ 1 Johann Bernoulli Institute for Mathematics and Computer Science, \\ University of Groningen, The Netherlands \\ 2 Dept. of Computer Eng. and Electrical Eng. and Applied Mathematics, \\ University of Salerno, Italy \\ 3 Intelligent Computer Systems, University of Malta, Malta \\ \{n.strisciuglio,g.azzopardi,n.petkov\}@rug.nl, mvento@unisa.it
}

\begin{abstract}
We propose a delineation algorithm that deals with bar-like structures of different thickness. Detection of linear structures is applicable to several fields ranging from medical images for segmentation of vessels to aerial images for delineation of roads or rivers. The proposed method is suited for any delineation problem and employs a set of $B$-COSFIRE filters selective for lines and lineendings of different thickness. We determine the most effective filters for the application at hand by Generalized Matrix Learning Vector Quantization (GMLVQ) algorithm. We demonstrate the effectiveness of the proposed method by applying it to the task of vessel segmentation in retinal images. We perform experiments on two benchmark data sets, namely DRIVE and STARE. The experimental results show that the proposed delineation algorithm is highly effective and efficient. It can be considered as a general framework for a delineation task in various applications.
\end{abstract}

\section{Introduction}

The automatic delineation of linear structures in images has gained interest in the image processing community, due to its applicability to several problems in different fields. In medical image analysis, for instance, the automatic segmentation of blood vessels from retinal fundus images, $\mathrm{x}$-ray fluoroscopy images or angiography images, among others, is a basic step before proceeding with further processing for the diagnosis of several pathologies. Other applications involve the quantification of length and width of cracks in walls (Fig. 1c) [17] for earthquake damage estimation or for monitoring the flow of rivers in order to prevent flooding disasters (Fig. 1d) [25].

In this paper we focus on the delineation of blood vessels in retinal fundus images (Fig.1a), which is a fundamental step for further analysis for the formulation of a diagnostic hypothesis. The manual inspection of such images is a time-consuming and expensive process. Therefore, mass screening is only possible if it is assisted by computer aided systems. The effective segmentation of the vascular tree and the background is very important because the tree contains significant information about the geometrical structure of the vessels; furthermore, any lesions in the background can be analysed better after the vessels are segmented. 


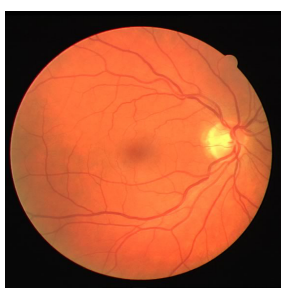

(a)

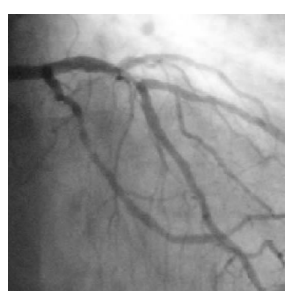

(b)

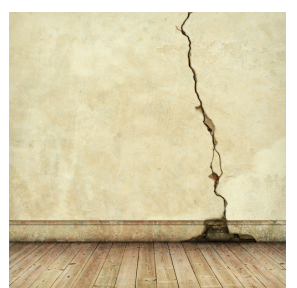

(c)

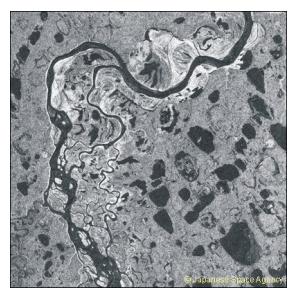

(d)

Fig. 1: Few examples where delineation algorithms are applicable: (a) blood vessels in a retinal fundus image, (b) x-ray fluoroscopy image, (c) cracks in walls and (d) a river in an aerial image.

Several methods have been proposed to automatically delineate the blood vessel tree. They can be generally divided into two categories: (i) non-supervised, based on filtering or template matching, and (ii) supervised, based on machine learning techniques.

Among the non-supervised methods, matched filtering techniques model the profile of a vessel by a 2D-Gaussian kernel $[6,9,1]$. Vessel tracking methods, instead, start from an initial set of points chosen either automatically or manually, to segment the vessels by following their center-line $[13,26,7]$. Other methods use mathematical morphology and a-priori knowledge on the vessel tree structure [24, 16]. In [15], a vessel growing procedure based on multiscale information about vessels' width, size and orientation is proposed. A multiconcavity modeling approach with differentiable concavity measure was proposed in [12].

Supervised methods share a common methodology: they treat the matter as a twoclass problem, form pixel-wise feature vectors by some feature extraction methods and learn a classification model based on vessel and non-vessel training feature vectors. The responses of multiscale Gaussian filter and ridge detectors have been used as pixelwise features together with a $k$-NN classifier in [18] and [23], respectively. In [22], a Bayesian classifier is combined with features obtained through multiscale analysis of Gabor wavelets. A rotation-invariant line operator in combination with a support vector machine (SVM) is proposed in [20], while in [14] a multilayer neural network has been applied to classify pixels based on moment-invariant features. An ensemble of bagged and boosted decision trees is employed in [8].

Supervised methods usually employ high-dimensional pixel-wise feature vectors and the choice of the features is usually influenced by domain knowledge. They are designed to overcome specific problems of retinal fundus images. In [8], for instance, morphological transformations and multiscale Gabor filters are used to eliminate bright and dark lesions, respectively.

We propose to address the problem of delineating bar-like structures (i.e. vessels) of different thickness by employing a set of $B$-COSFIRE filters of the type proposed in [5], configured to be selective for vessels of different thickness. In [5], a bar detector and a bar-ending detector have been combined by summing up their responses. The configuration parameters of the line-ending detector have been determined to complement the performance of a line detector. This implies a chain of dependency in the construction 


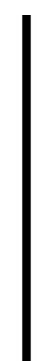

(a)

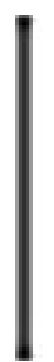

(b)

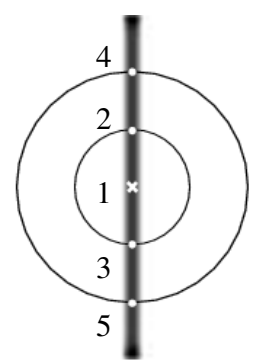

(c)

Fig. 2: Configuration of a $B$-COSFIRE filter. (a) A vertical line is processed by (b) a $D o G$ filter. Then, (c) the positions of strong $D o G$ responses along a set of concentric circles around the interest point (labeled with ' 1 ') are determined.

of the filters whose complexity increases with increasing number of filters. In this work, instead, we determine a subset of $B$-COSFIRE filters by means of a learning process. In particular, we use the Generalized Matrix Learning Vector Quantization (GMLVQ) method that determines a weight called relevance for each filter and we select the ones with the highest relevances.

The rest of the paper is organized as follows: in Section 2 we explain the proposed approach for filter selection. In Section 3 we present the experimental results. After a discussion of the results and comparison with the state of the art methods in Section 4, we draw conclusions in Section 5.

\section{Method}

The proposed delineation algorithm is based on automatic selection of the $B$-COSFIRE filters with the highest relevances. We configure a set of $B$-COSFIRE filters selective for lines of different thickness (i.e. scale). Instead of manually setting the scale of the filters, we determine them by means of a learning process. Such procedure allows to select those filters that give highest contribution to the delineation task.

\subsection{B-COSFIRE filter}

A $B$-COSFIRE filter, originally proposed in [5], takes as input the responses of a group of Difference-of-Gaussians $(D o G)$ filters at certain positions with respect to the center of its area of support. Such positions are determined in an automatic configuration process performed on a prototype pattern, Fig. 2. We use a synthetic line as prototype pattern (Fig. 2a) and process it with a $D o G$ filter (Fig. 2b). We consider the $D o G$ filter responses along a number of concentric circles around the center point (labeled with ' 1 ' in Fig. 2c). The result of the configuration is a set of 3 -tuples: $S=\left\{\left(\sigma_{i}, \rho_{i}, \phi_{i}\right) \mid i=1, \ldots, n\right\}$, where $\sigma_{i}$ represents the standard deviation of the outer $D o G$ function ${ }^{4}$, while $\left(\rho_{i}, \phi_{i}\right)$ are the polar coordinates of the $i$-th $D o G$ response with respect to the support center of the filter.

\footnotetext{
${ }^{4}$ The standard deviation of the inner Gaussian function is $0.5 \sigma_{i}$
} 


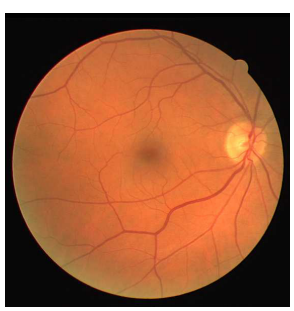

(a)

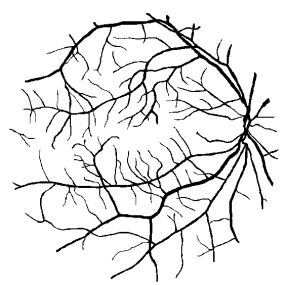

(e)

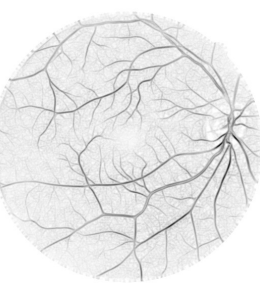

(b)

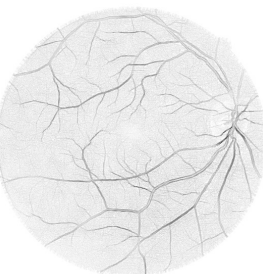

(f)

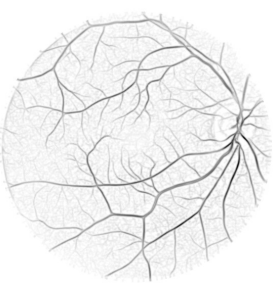

(c)

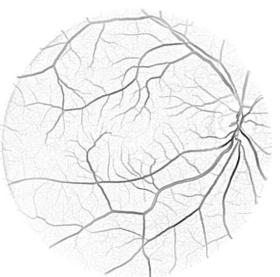

$(\mathrm{g})$

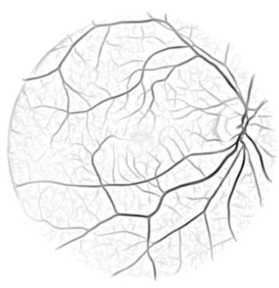

(d)

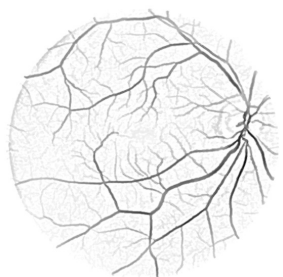

(h)

Fig. 3: (a) A retinal fundus image from the DRIVE data set and (e) its segmented vessel tree (used as a ground truth). In the second column the responses of small-scale (b) line and (f) line-ending filters are depicted. In the third and forth columns, the responses of $(\mathrm{c}, \mathrm{g})$ medium-scale and $(\mathrm{d}, \mathrm{h})$ large-scale filters are shown, respectively.

We compute the output of a $B$-COSFIRE filter as the geometric mean of the responses of the concerned $D o G$ filters at the positions determined in the configuration step:

$$
r_{S}(x, y) \stackrel{\text { def }}{=}\left(\prod_{i=1}^{|S|}\left(s_{\sigma_{i}, \rho_{i}, \phi_{i}}(x, y)\right)\right)^{1 /|S|}
$$

where $s_{\sigma_{i}, \rho_{i}, \phi_{i}}(x, y)$ is the blurred $D o G$ filter response with scale $\sigma_{i}$. We blur the $i$ th $D o G$ response by taking the maximum response in a local neighborhood weighted by a Gaussian function centered in $\left(\rho_{i}, \phi_{i}\right)$. The standard deviation of such Gaussian function is a linear function of the distance $\rho_{i}: \sigma^{\prime}=\sigma_{0}^{\prime}+\alpha \rho_{i}$.

In order to achieve rotation-tolerance, we first manipulate the parameter $\phi_{i}$, obtaining a new set $R_{\psi}(S)=\left\{\left(\sigma_{i}, \rho_{i}, \phi_{i}+\psi\right) \mid i=1, \ldots, n\right\}$ with orientation preference $\psi$. Then, we take the maximum across the responses of $B$-COSFIRE filters selective for different orientation preferences:

$$
\hat{r}_{S}(x, y) \stackrel{\text { def }}{=} \max _{\psi \in \Psi}\left\{r_{R_{\psi}(S)}(x, y)\right\}
$$

where $\Psi=\left\{\frac{\pi i}{12} \mid i=0 \ldots 11\right\}$. In this work, we use the publicly available Matlab implementation of a $B$-COSFIRE filter ${ }^{5}$.

\footnotetext{
${ }^{5}$ http://www.mathworks.com/matlabcentral/fileexchange/49172
} 


\subsection{A bank of $B$-COSFIRE filters}

To overcome the intrinsic delineation error at bar-endings due to the multiplicative character of $B$-COSFIRE filters, we employ two kinds of filter: line and line-ending detector. In order to also account for the different thickness of vessels in a given image we design a bank of 21 line $\left(\left\{S_{1}, \ldots S_{21}\right\}\right)$ and 21 line-ending $\left(\left\{S_{22}, \ldots S_{42}\right\}\right) B$-COSFIRE filters of varying scale $\sigma$.

As an example, in Fig. 3 we show the responses of line and line-ending $B$-COSFIRE filters selective for thin (second column), medium (third column) and thick (forth column) vessels. It is evident how the small-scale filters respond better along thin vessels (Fig. 3b and Fig. 3f) but are more sensitive to background noise. Large-scale filters, instead, show higher responses along thicker vessels (Fig. 3d and Fig. 3h) and are more robust to noise.

In this way, we characterize a pixel at location $(x, y)$ by the 42 responses of $B$ COSFIRE filters plus the intensity value of the green channel of the RGB image (further details about this choice are given in Section 3.2), which results in a 43-element feature vector denoted by $v(x, y)$ :

$$
v(x, y)=\left[G(x, y), \hat{r}_{S_{1}}(x, y), \ldots, \hat{r}_{S_{42}}(x, y)\right]^{T}
$$

where $G(x, y)$ is the intensity value of the green channel and $\hat{r}_{S_{i}}(x, y)$ is the response of a $B$-COSFIRE filter $S_{i}$.

\subsection{Filters selection}

We use the Generalized Matrix LVQ algorithm (GMLVQ) [21] in order to select filters. The GMLVQ is a supervised prototype-based learning algorithm that, given a feature space, determines the most representative prototypes for each class in the data set. Besides learning the prototypes, it computes the individual and pair-wise relevances of all involved features in the distance metric.

We form feature vectors composed by the responses of a bank of $B$-COSFIRE filters together with the classes of the corresponding pixels (vessel or background) as input to the GMLVQ algorithm. Next, GMLVQ computes a full matrix $(43 \times 43)$ of the relevances of all pairs of filters. The values on the diagonal of the resulting matrix, depicted in Fig 4, give an indication of the most relevant filters for the classification problem at hand. The higher the coefficient the more relevant that filter is in comparison to the others. In this work, we consider the relevances of the single $B$-COSFIRE filters, and select only the ones that achieve local relevance maximum along the space dimension, Fig 4 . The dimensionality of the initial feature vectors is thus reduced and the overall processing time is substantially improved.

\subsection{Classification}

Finally, we use the training feature vectors composed of the responses of the selected filters to train a SVM classifier with a linear kernel, which is particularly suited for binary classification problems and has high generalization capabilities [10]. 


\section{Experimental results}

\subsection{Data sets}

We evaluate the proposed method on the benchmark data sets DRIVE [23] and STARE [9], that come with manually segmented images to be used as ground truth.

The DRIVE data set contains 40 images (of size $565 \times 584$ pixels), equally divided into a training and a test set. The images in the training set are manually segmented by one human observer, while the images in the test set are segmented by two other observers. The second observer segmentation is normally used to compute the human performance results. All the images come with a binary mask that indicate the field of view (FOV). The STARE data set consists of 20 color retinal fundus images (of size $700 \times 605$ pixels), 10 of which contain signs of pathologies. Each image is manually segmented by two different observers.

We measure the performance of our method by comparing the automatically generated binary images with the ground truth provided by the first observer.

\subsection{Preprocessing}

We only consider the green channel of the color retinal images because it shows the highest contrast between vessels and background $[23,16]$. In order to smoothen the high contrast along the border of the field of view (FOV) of the retina we employ the preprocessing algorithm proposed in [22]. It consists in a procedure that iteratively enlarges the region of interest delimited by the FOV. Finally, we enhance the image by using the contrast-limited adaptive histogram equalization (CLAHE) algorithm [19].

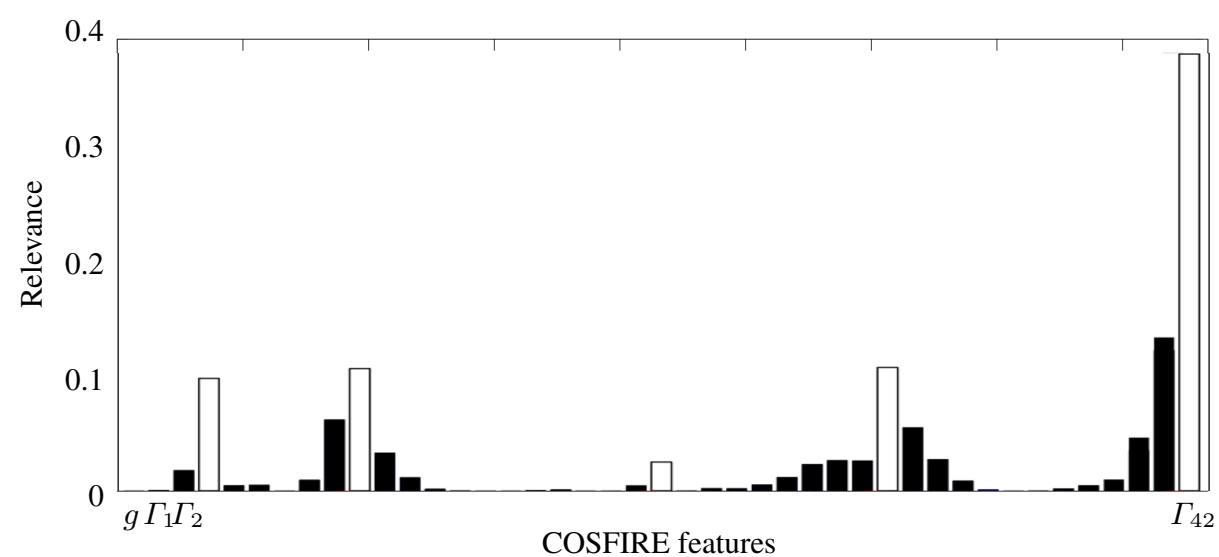

Fig. 4: The relevances of the features as obtained by the GMLVQ algorithm for the training images of the DRIVE data set. The local maxima of the relevances (white bars) represent the filters selected for classification. 


\subsection{Training}

For the DRIVE data set we randomly select 20000 vessel pixels (1000 from each image) and 20000 non-vessel pixels (1000 from each image) from the images in the training set. The STARE data set does not have separate training and test sets. Thus, we construct the training set by randomly choosing 40000 pixels from all the 20 images in the data set (1000 vessel pixels and 1000 non-vessel pixels from each image). As suggested in $[20,8]$, since the size of the selected training set is very small $(<0.5 \%$ of the entire data set), we evaluate the performance on the whole set of images.

For each pixel we generate a 43 -element feature vector as described in Section 2.2. We configure the line-selective $B$-COSFIRE filters with the following $\sigma$ values: $\{1.4+$ $0.1 j \mid 0 \leq j \leq 20\}$ for DRIVE and $\{1.7+0.1 j \mid 0 \leq j \leq 20\}$ for STARE. For the lineending-selective $B$-COSFIRE filters we use the following $\sigma$ values: $\{0.9+0.1 j \mid 0 \leq$ $j \leq 20\}$ for DRIVE and $\{1.4+0.1 j \mid 0 \leq j \leq 20\}$ for STARE.

In order to reduce the skewness in the data, for each element $v_{i}$ in a feature vector we apply the inverse hyperbolic sine transformation function [11], defined as:

$$
f\left(v_{i}, \theta\right)=\sinh ^{-1}\left(\theta v_{i}\right) / \theta
$$

For large values of $v_{i}$ and $\theta>0$ it behaves like a $\log$ transformation $^{6}$. As $\theta \rightarrow 0$, $f\left(v_{i}, \theta\right) \rightarrow v_{i}$.

We then use the resulting feature vectors and their class labels as input to the GMLVQ algorithm, which determines the relevance of each filter. The bar plot in Fig. 4 shows the relevances $\Gamma_{i}$ of every $B$-COSFIRE filter used for the training images in the DRIVE data set. We select the filters whose responses correspond to local maxima in the relevance landscape to form the feature vectors. For the DRIVE data set, we select three line (at scales $\sigma_{1}=1.6, \sigma_{2}=2.2$ and $\sigma_{3}=3.4$ ) and two line-ending (at scales $\sigma_{4}=1.7$ and $\left.\sigma_{5}=2.9\right) B$-COSFIRE filters. For the STARE data set, instead, we select two line $\left(\sigma_{1}=3\right.$ and $\left.\sigma_{2}=3.7\right)$ and three line-ending $\left(\sigma_{3}=2, \sigma_{4}=2.7\right.$ and $\left.\sigma_{5}=3.4\right)$ $B$-COSFIRE filters. For both data sets, we discard the pixel value of the green channel, since it has very low relevance. In this way the final representation results in a 5-element vector, for both data sets. It is worth noting that the different values of $\sigma$ automatically selected for the two data sets depend on the different resolution and thickness of the vessels in the images in such data sets.

\subsection{Evaluation and results}

The output of the SVM classifier is a probability score that indicates the vesselness of each pixel: 1 and 0 represent a vessel pixel and a background pixel with absolute certainty, respectively. We threshold the output of the SVM classifier to obtain the final binary vessel map. The threshold divides the pixels into two classes: vessel and nonvessel pixels. We compare the resulting binary map with the corresponding ground truth and compute the following measures: true positives (TP), false positives (FP), true negatives (TN) and false negatives (FN). We consider only the pixels inside the FOV for

\footnotetext{
${ }^{6}$ The value of $\theta$ has been experimentally determined on some training images and set to 1000 for both the DRIVE and STARE data sets.
} 
DRIVE

STARE

\begin{tabular}{|c|c|c|c|c|}
\hline & [5] & Proposed method & [5] & Proposed method \\
\hline Accuracy & 0.9442 & 0.9467 & 0.9497 & 0.9537 \\
\hline AUC & 0.9616 & 0.9588 & 0.9563 & 0.9629 \\
\hline Specificity & 0.9704 & 0.9724 & 0.9701 & 0.9717 \\
\hline Sensitivity & 0.7655 & 0.7731 & 0.7716 & 0.8011 \\
\hline $\mathrm{MCC}$ & 0.7475 & 0.7566 & 0.7335 & 0.7452 \\
\hline Processing time & $10 \mathrm{~s}$ & $60 \mathrm{~s}$ & $10 \mathrm{~s}$ & $60 \mathrm{~s}$ \\
\hline
\end{tabular}

Table 1: Experimental results achieved by the proposed supervised method on the DRIVE and STARE data sets in comparison to the ones achieved in [5] by using unsupervised $B$-COSFIRE filters. The average processing time is also reported.

the evaluation. In order to compare the performance of the proposed method with other state of the art algorithms, we calculate the accuracy (Acc), sensitivity (Se), specificity (Sp) and Matthews correlation coefficient (MCC). These metrics are defined as follows:

$$
\begin{gathered}
A c c=\frac{T P+T N}{N}, S e=\frac{T P}{T P+F N}, S p=\frac{T N}{T N+F P}, \\
M C C=\frac{T P / N-S \times P}{\sqrt{P \times S \times(1-S) \times(1-P)}},
\end{gathered}
$$

where $N=T N+T P+F N+F P, S=(T P+F N) / N$ and $P=(T P+F P) / N$.

The MCC measures the quality of a binary classification, taking into account the unbalanced cardinality of the two classes. The MCC value varies from -1 for a completely wrong prediction to 1 for a perfect classification system. A value of 0 corresponds to random prediction.

We compare the performance of the proposed method with others by also computing the receiving operator characteristics (ROC) curve and its underlying area (AUC). It allows the evaluation of the overall performance of the algorithms by considering the trade-off between sensitivity and specificity. The closer the ROC curve to the top-left corner, the better the classification is. In the case of a perfect system the ROC curve has a point $(0,1)$.

We compute the sensitivity, specificity and accuracy for each data set for a specific value of threshold $t$, the one that contributes to the maximum average MCC value on the corresponding data set. In Table 1 we report the results achieved by the proposed supervised method on the DRIVE and STARE data sets, compared with the ones obtained by the original unsupervised approach that used $B$-COSFIRE filters [5]. The performance improvement is attributable to the learning ability of combining the responses of $B$-COSFIRE filters that are selective for tiny and large vessels, as well as for vessel-endings. 


\section{Discussion}

The performance results that we achieve on the DRIVE and STARE data sets are better than many of other methods and confirm the effectiveness of the proposed method (Table 2). For comparison purposes, we move along the ROC curve and compare the performance of the proposed method with the ones of other supervised methods. For the STARE data set and for the same specificity values reported in [22], [14] and [8] ( $S p=0.9747, S p=0.9819$ and $S p=0.9763$ ) we achieve better sensitivity: 0.7806, 0.7316 and 07697 , respectively. Similarly, for the DRIVE data set and for the same specificity reported in [22] $(S p=0.9782)$ and in [14] $(S p=0.9801)$ we achieve better sensitivity: 0.7425 and 0.7183 , respectively. For the same specificity reported in [8] $(S p=9807)$ we achieve a lower value of the sensitivity $(S e=0.7181)$.

The main contribution of the proposed method concerns the features extraction and selection procedure. Indeed, the employed filters are domain-independent. The proposed algorithm can be considered as a general framework for the delineation of any bar-like structures. Moreover, the filter selection process by GMLVQ is very effective to choose a small subset of filters that give a significant contribution. This generalization ability is attributable to the trainable character of $B$-COSFIRE filters, in that they are automatically configured by some vessel-like prototype patterns. In contrast, other methods use hand-crafted features which require domain knowledge. For instance, the features proposed in [8] are specifically designed to deal with particular issues of the retinal fundus images, such as bright and dark lesions or non-uniform illumination of the FOV.

The feature set may also be expanded by adding the responses of filters selective for other kinds of patterns such as bifurcations and crossovers [3, 2]. In future we aim to investigate the addition of the inhibition mechanism proposed in [4], which significantly improves signal-to-noise ratio in contour detection.

Besides achieving high effectiveness, the proposed methodology is also very efficient. In practice, an image from the DRIVE $(565 \times 584$ pixels $)$ or the STARE $(700 \times 605$ pixels) data set is processed (preprocessing and feature extraction followed by classification and thresholding) in less than 60 seconds, which is faster than all the other supervised approaches (15 minutes [23], 3 minutes [22], 1.5 minutes [14], 2 minutes [8]). We measured this duration by running a sequential Matlab implementation on a notebook with a $1.8 \mathrm{GHz}$ processor. Since the $B$-COSFIRE feature extraction method relies on independent computations, the proposed methodology can become even faster by a parallel implementation.

\section{Conclusion}

The delineation method that we propose is highly effective and efficient for the segmentation of vessel trees in retinal fundus images. In particular, this method is very effective in the detection of tiny vessels. The results that we achieve on two publicly available benchmark data sets, DRIVE $(\mathrm{Se}=0.7731, \mathrm{Sp}=0.9724)$ and STARE $(\mathrm{Se}=0.8011$, $\mathrm{Sp}=0.9717)$ are comparable with many of the existing methods, while showing higher time efficiency. 
The proposed methodology can be considered as a general delineation framework and could be used in other applications that require the segmentation of vessel-like structures. This is mainly attributable to the trainable $B$-COSFIRE filters coupled with the automatic feature selection performed by GMLVQ. 


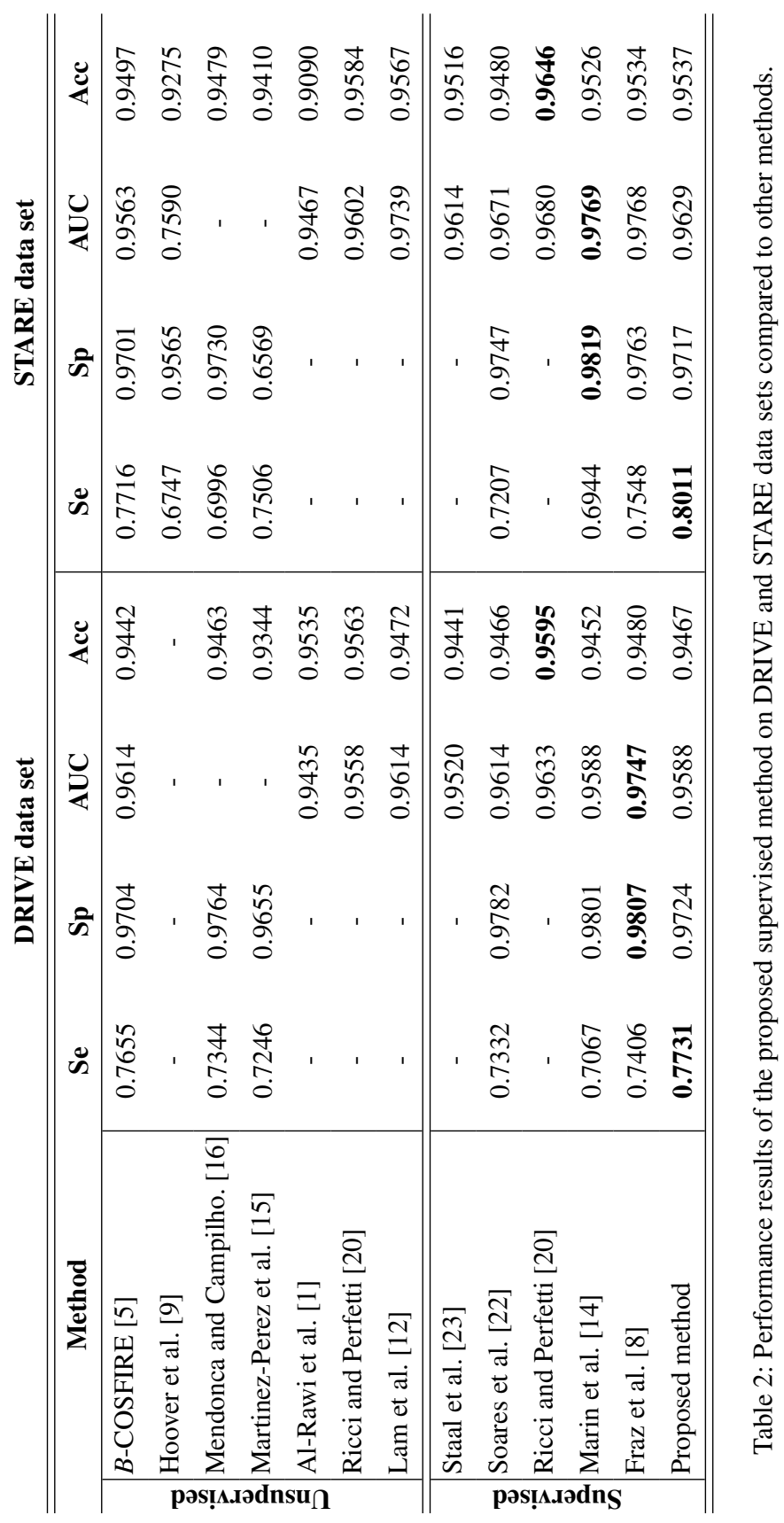




\section{Bibliography}

[1] Al-Rawi, M., Qutaishat, M., Arrar, M.: An improved matched filter for blood vessel detection of digital retinal images. Computer in biology and medicine 37(2), 262-267 (2007)

[2] Azzopardi, G., Petkov, N.: Automatic detection of vascular bifurcations in segmented retinal images using trainable COSFIRE filters. Pattern Recognition Letters 34, 922-933 (2013)

[3] Azzopardi, G., Petkov, N.: Trainable COSFIRE filters for keypoint detection and pattern recognition. IEEE Transactions on Pattern Analysis and Machine Intelligence 35, 490-503 (2013)

[4] Azzopardi, G., Rodríguez-Sánchez, A., Piater, J., Petkov, N.: A push-pull CORF model of a simple cell with antiphase inhibition improves SNR and contour detection. PLoS ONE 9(7), e98424 (07 2014)

[5] Azzopardi, G., Strisciuglio, N., Vento, M., Petkov, N.: Trainable COSFIRE filters for vessel delineation with application to retinal images. Medical Image Analysis 19(1), 46 - 57 (2015)

[6] Chauduri, S., Chatterjee, S., Katz, N., Nelson, M., Goldbaum, M.: Detection of blood-vessels in retinal images using two-dimensional matched-filters. IEEE Transactions on medical imaging 8(3), 263-269 (1989)

[7] Chutatape, O., Liu Zheng, Krishnan, S.: Retinal blood vessel detection and tracking by matched Gaussian and Kalman filters. In: Chang, H., Zhang, Y. (eds.) Proc. 20th Annu. Int. Conf. IEEE Eng. Med. Biol. Soc. (EMBS'98). vol. 17, pp. 3144-9 (1998)

[8] Fraz, M., Remagnino, P., Hoppe, A., Uyyanonvara, B., Rudnicka, A., Owen, C., Barman, S.: An ensemble classification-based approach applied to retinal blood vessel segmentation. IEEE Transactions on Biomedical Engineering 59(9), 25382548 (2012)

[9] Hoover, A., Kouznetsova, V., Goldbaum, M.: Locating blood vessels in retinal images by piecewise threshold probing of a matched filter response. IEEE Transactions on medical imaging 19(3), 203-210 (2000)

[10] Joachims, T.: Estimating the generalization performance of an svm efficiently. In: Proceedings of the Seventeenth International Conference on Machine Learning. pp. 431-438. ICML '00, Morgan Kaufmann Publishers Inc., San Francisco, CA, USA (2000)

[11] Johnson, N.L.: Systems of frequency curves generated by methods of translation. Biometrika 36(1-2), 149-176 (1949)

[12] Lam, B., Gao, Y., Liew, A.C.: General retinal vessel segmentation using regularization-based multiconcavity modeling. IEEE Transactions on Medical Imaging 29(7), 1369-1381 (2010)

[13] Liu, I., Sun, Y.: Recursive tracking of vascular networks in angiograms based on the detection deletion scheme. IEEE Transactions on medical imaging 12(2), 334 341 (1993) 
[14] Marin, D., Aquino, A., Emilio Gegundez-Arias, M., Manuel Bravo, J.: A New Supervised Method for Blood Vessel Segmentation in Retinal Images by Using Gray-Level and Moment Invariants-Based Features. IEEE Transactions on medical imaging 30(1), 146-158 (2011)

[15] Martinez-Pérez, M.E., Hughes, A.D., Thom, S.A., Bharath, A.A., Parker, K.H.: Segmentation of blood vessels from red-free and fluorescein retinal images. Medical Image Analysis 11(1), 47-61 (2007)

[16] Mendonca, A.M., Campilho, A.: Segmentation of retinal blood vessels by combining the detection of centerlines and morphological reconstruction. IEEE Transactions on Medical Imaging 25(9), 1200-1213 (2006)

[17] Muduli, P., Pati, U.: A novel technique for wall crack detection using image fusion. In: Computer Communication and Informatics (ICCCI), 2013 International Conference on. pp. 1-6 (Jan 2013)

[18] Niemeijer, M., Staal, J., van Ginneken, B., Loog, M., Abramoff, M.: Comparative study of retinal vessel segmentation methods on a new publicly available database. In: Proc. of the SPIE - The International Society for Optical Engineering. pp. 64856 (2004), Medical Imaging 2004. Image Processing, 16-19 Feb. 2004, San Diego, CA, USA

[19] Pizer, S., Amburn, E., Austin, J., Cromartie, R., Geselowitz, A., Greer, T., Ter Haar Romeny, B., Zimmerman, J., Zuiderveld, K.: Adaptative Histogram Equalization and its Varations. Computer Vision Graphics and Image Processing 39(3), 355-368 (1987)

[20] Ricci, E., Perfetti, R.: Retinal blood vessel segmentation using line operators and support vector classification. IEEE Transactions on medical imaging 26(10), 1357-1365 (2007)

[21] Schneider, P., Biehl, M., Hammer, B.: Adaptive relevance matrices in learning vector quantization. Neural Comput. 21(12), 3532-3561 (Dec 2009)

[22] Soares, J.V.B., Leandro, J.J.G., Cesar, Jr., R.M., Jelinek, H.F., Cree, M.J.: Retinal vessel segmentation using the 2-D Gabor wavelet and supervised classification. IEEE Transactions on medical imaging 25(9), 1214-1222 (2006)

[23] Staal, J., Abramoff, M., Niemeijer, M., Viergever, M., van Ginneken, B.: Ridgebased vessel segmentation in color images of the retina. IEEE Transactions on medical imaging 23(4), 501-509 (2004)

[24] Zana, F., Klein, J.: Segmentation of vessel-like patterns using mathematical morphology and curvature evaluation. IEEE Transactions on medical imaging 10(7), 1010-1019 (2001)

[25] Zhang, L., Zhang, Y., Wang, M., Li, Y.: Adaptive river segmentation in sar images. Journal of Electronics (China) 26(4), 438-442 (2009)

[26] Zhou, L., Rzeszotarski, M., Singerman, L., Chokreff, J.: The detection and quantification of retinopathy using digital angiograms. IEEE Transactions on medical imaging 13(4), 619-626 (1994) 